\title{
El cooperativismo en la escuela rural del México de los años treinta/
}

\section{The Cooperativism in the Mexican Rural School, 1930s}

\author{
Alicia Civera Cerecedo \\ El Colegio Mexiquense, \\ Zinacantepec, México
}

Después de la Revolución de 1910 el gobierno mexicano impulsó un sistema de enseñanza agrícola que pretendía apoyar el desarrollo de la producción agropecuaria y mejorar las condiciones de vida de los campesinos. En la década de los treinta se enlazó más fuertemente la escuela con el desarrollo económico rural. La enseñanza agrícola, basada en el cooperativismo, no fue muy exitosa pero proporcionó apoyo a la economía familiar basada en la producción de autoconsumo dentro del sistema capitalista, mientras que el cooperativismo enseñado en la escuela sirvió más como base de asociación política que como estrategia económica.

PALABRAS CLAVE: Escuela rural; Desarrollo rural; Cooperativismo; Enseñanza agropecuaria.

After the 1910 Revolution, the Mexican government promoted an agricultural education system to develop agricultural production and improve peasant's way of life. During the 1930s, the school was strongly involved in economic development. The agricultural education - based on cooperativism - wasn't very successful but was helpful to improve familiar auto-production economy inside the capitalism system. Meanwhile, cooperativism at school was more a kind of political association than an economic strategy.

KEYwords: Rural School; Rural Development; Cooperativism; Agricultural Education. 


\section{Introducción}

Pese a una creciente bibliografía sobre la educación rural en el México posrevolucionario, es poco lo que sabemos acerca de los alcances de la enseñanza agrícola y sobre todo del cooperativismo en el campo mexicano. Como en otros países de América Latina, en México se intentó enlazar la obra educativa con el impulso del desarrollo económico en las zonas rurales pero, a diferencia de otras experiencias, este vínculo tendría características peculiares al desarrollarse tras la Revolución de 1910 y las modificaciones que trajo consigo la reforma agraria y la conformación de un nuevo Estado.

Si bien desde el siglo XIX hubo algunas experiencias de enseñanza agrícola, enfocada sobre todo a la modernización de la producción en las grandes propiedades, a partir del triunfo de la Revolución comenzó a cobrar fuerza la necesidad de organizar un sistema de enseñanza agrícola de carácter popular. Junto con los maestros, los agrónomos formaron parte de los intelectuales que articularon una política para transformar la mente y la vida de los campesinos. Los maestros se plantearon el reto de expandir la escuela por todo el país y que ésta sirviera para mejorar la vida de los campesinos, mientras que los agrónomos pusieron sus conocimientos al servicio del nuevo Estado revolucionario, que era prácticamente el único actor capaz de emplearlos para planear la reforma agraria y promover el desarrollo de los pequeños propietarios o de los nuevos ejidos en un momento en que la inversión extranjera se contrajo a raíz de los diez años de guerra y en el que los grandes propietarios se sentían amenazados por la reforma agraria.

La labor cultural, económica y política de los maestros y los agrónomos, sin embargo, no siempre fue armoniosa al tener diferencias importantes en sus culturas profesionales e ideologías y durante los años veinte se realizó fundamentalmente desde dos instancias distintas: la Secretaría de Educación Pública (SEP) y la Secretaría de Agricultura y Fomento (SAF), con proyectos distintos, pero estrategias similares. La SEP inició la incorporación de la enseñanza agrícola dentro del currículum de la escuela rural y normal rural, a partir sobre todo de la construcción de anexos productivos en donde los estudiantes aprenderían de manera práctica y a partir de fomentar el cooperativismo. Asimismo, introdujo la enseñanza de aspectos agrícolas y de oficios rurales en las misiones culturales que viajaban por todo el país. La SAF construyó ocho escuelas centrales agrícolas para for- 
mar expertos en la producción agrícola y la creación de cooperativas de producción, por un lado, y por el otro a partir de un sistema de agrónomos regionales que viajaban por todo el territorio dando asesorías a productores de todo tipo. ${ }^{1}$

Pero el vínculo entre la promoción del desarrollo económico rural y la escuela se fortaleció de manera importante durante la década de los años treinta, a través del impulso al cooperativismo que se desplegó desde el Departamento de Enseñanza Agrícola y Normal Rural (DEANR), dentro de la SEP. En este artículo, mi intención es explicar en qué consistía el cooperativismo que trataba de enseñarse en la escuela rural, a través del examen de la forma en que se manejaban las cooperativas de consumo y producción de las escuelas regionales campesinas.

\section{Las escuelas regionales campesinas y el desarrollo integral del campo}

Las orientaciones de la política educativa, iniciada después de la Revolución de 1910 y la creación de la SEP, se fueron modificando a principios de la década de 1930 para dar un mayor peso a los aspectos de desarrollo económico, y entre ellos a la enseñanza agrícola y de oficios rurales, sobre todo a partir de que Narciso Bassols fuera secretario de Educación Pública (1931-1934). Desde su perspectiva y la de muchos maestros y agrónomos que se fueron radicalizando durante la crisis económica del 1929 y su acercamiento cotidiano a la vida de los campesinos, el campo mexicano sólo saldría adelante si había una transformación integral que se basara en la actividad económica y por ello buscaron colocar a la escuela rural en el centro de dicha transformación. Los cambios en los sistemas de producción y comercialización, sin embargo, dependían de la forma en que los campesinos se organizaran, así como de la adaptación o creación de técnicas apropiadas a las características de cada región y no de la importación de conocimientos generados en realidades ajenas a las del país. A la labor cultural, social, educativa y económica que los maestros rurales debían realizar, se incorporó una más: la política. La escuela debía guiar la lucha por la tierra, apoyar las gestiones para solicitar créditos y la defensa de los productores en contra de los acaparadores e intermediarios

1 Véase SAF, 1926; SEP, 1928a; SEP, 1928b; Cotter, 2003 y Loyo, 1999. 
a partir del cooperativismo. Fomentar este era, necesariamente, involucrarse en la política.

La intervención de los agrónomos en los asuntos de la escuela rural, por un lado, y la de los maestros rurales en la organización de los campesinos, por el otro, se intensificarían aún más durante la presidencia de Lázaro Cárdenas (1934-1940), cuando el reparto de tierras obtuvo un ritmo sin precedente. ${ }^{2}$ El reparto se vinculó con la reforma del artículo tercero constitucional aprobada en 1934, estipulando que la educación que impartiera el Estado sería socialista para formar en el educando una concepción científica y exacta del universo. Según el "Plan de acción de la educación socialista" que emitió la SEP para normar el trabajo de las escuelas primarias rurales, semiurbanas y urbanas, la escuela debía ser obligatoria y gratuita, de asistencia infantil, única, coeducativa, integral, vitalista, progresiva, científica, desfanatizante, orientadora, de trabajo, cooperativista, emancipadora y mexicana. ${ }^{3}$

Sin restar importancia a la reforma educativa cardenista, es necesario destacar que la acción educativa de la SEP en el campo ya había sufrido un cambio de trascendencia a partir de 1931, cuando se creó dentro de ella el DEANR que, desde entonces hasta su desaparición en 1941, fue encabezado por ingenieros agrónomos. De él dependieron las escuelas normales rurales y las misiones culturales, y de él emanaban las normativas sobre enseñanza agrícola y cooperativismo dirigidas a las escuelas primarias, secundarias, normales, técnicas, rurales, urbanas, etc. En 1932, el DEANR logró que las escuelas centrales agrícolas pasaran también a la SEP. Un año después se modificó la composición de las misiones culturales, que desde entonces estarían formadas por un jefe (que era maestro normalista), un maestro de educación física, uno de música, otro de artes plásticas y un enfermero; en lugar de una trabajadora social se integró una agente organizadora rural, y en lugar de un agrónomo un agente organizador rural. Con ayuda de material fílmico, los agentes organizadores rurales trabajaban con hombres y mujeres para instalar agua potable y baños, servicios postales, vigilar la higiene en mercados, rastros, escuelas y casa, realizar campañas antialcohólicas, promover la producción agrícola e industrial y, sobre todo,

2 Entre 1915 y 1934 se habían repartido poco más de 10.000 .000 de hectáreas a cerca de 1 millón de ejidatarios, mientras que entre 1935 y 1940 se repartieron casi 18.000 .000 hectáreas a 772.000 ejidatarios. De todos modos, el número de beneficiarios parece pequeño si se considera que en 1940 el 78.1\% de la población total del país vivía en poblaciones rurales (más de 15 millones de habitantes) y el $63.3 \%$ de la población económicamente activa se dedicaba a la agricultura. Bartra, 1985.

3 García Téllez, 1935, pp. 227-238; SEP, 1935, pp. 176-177. 
la tramitación de créditos, arreglar problemas de agua o fomentar proyectos de riego, incluyendo la forma en que debían organizarse los campesinos para trabajar y vender sus productos. ${ }^{4}$

En las escuelas centrales agrícolas se intentó volver al proyecto original y matizar las múltiples contradicciones por las que habían pasado en una actividad aún indefinida entre el fomento de la agricultura para grandes propiedades, la modernización de pequeñas propiedades y el impulso al ejido, entre muchas otras. Ante las críticas externas y los movimientos de protesta de los propios estudiantes, se pusieron contrapesos al poder de los directores, varios de cuales habían sido acusados de malgastar o desviar los recursos de las escuelas, o de someter a los estudiantes a un trabajo excesivo. En lugar de algunos de ellos, se fue contratando a una nueva generación de agrónomos más radicales que buscaban afianzar el papel de la agronomía como una ciencia que, al servicio del Estado, aportaría el saber necesario para alcanzar algún día una sociedad sin clases, y que, por lo tanto, estaban más comprometidos con un proyecto popular. ${ }^{5}$

En 1932 el ingeniero Manuel Mesa Andraca, jefe del DEANR y mano derecha de Bassols, realizó una evaluación de la acción educativa en el campo y después de hacer una fuerte crítica a la pobreza en que vivían las 17 escuelas normales rurales que trabajaban en el país, la escasa influencia que podían ejercer las misiones culturales al ser tan pasajera su presencia en las comunidades y las contradicciones de las escuelas centrales agrícolas, enlazó a estas tres instituciones en una sola: las escuelas regionales campesinas. Las nuevas escuelas que comenzaron a fusionarse o crearse desde 1932 debían trabajar con un plan de estudios de cuatro años. Los dos primeros se dedicarían a la formación de técnicos agrícolas y aunque se mantenía la idea de propiciar mejores técnicas de producción y la promoción de industrias y oficios rurales que ayudaran al sostenimiento familiar, se ponía énfasis en partir del conocimiento de la situación específica de cada región en lugar de la importación ciega de técnicas extranjeras y la uti-

4 Archivo Histórico de la Secretaría de Educación Pública (AHSEP), Departamento de Enseñanza Agrícola y Normal Rural (DEANR), Informes de las Misiones Culturales, 1932-1933; SEP, 1933a, p. 227. La agente organizadora rural trabajaba con las mujeres en acciones que repercutían en la economía familiar o comunitaria, con funciones más amplias que las trabajadoras sociales, que trataban sobre todo cuestiones higiénicas. De todos modos, el trabajo de los organizadores sociales estaba diferenciado por género. Véase como ejemplo Civera, 1997, pp. 89-132.

5 AHSEP, DEANR, 164.9, Informes de la Escuela de Tenería, 1932-1933; ibidem, 969/10, Tamatán, Reglamento Interior, febrero de 1934; LAS, 1935; LAS, s/f; LAS, 1936; Loyo, 2004, p. 95; Bassols, 1964, pp. 53-56; Civera, 2008, pp. 121-137. 
lización de maquinaria inaccesible para los campesinos. Así mismo, se introducirían materias sobre "organización rural" y "economía doméstica" 6 - su equivalente para las mujeres - con el fin de que los estudiantes aprendieran métodos "para ganarse la confianza de la gente y promover el desarrollo económico y cultural".

Al terminar dicha formación, podría escogerse a los mejores estudiantes para seguir sus estudios otros dos años hasta titularse como maestros rurales, durante los cuales se cursarían materias de cultura general y pedagógica como en las normales rurales pero con mayor intensidad y se desarrollarían actividades de ganadería, agricultura, oficios e industrias rurales. La idea era que el nuevo maestro rural fuese "un ejidatario modelo". La SEP dispuso que los estudiantes fueran campesinos, de preferencia ejidatarios, pues a ellos debían beneficiar las acciones de la escuela y ellos, como campesinos, entenderían los problemas del medio rural. Como se buscaban alumnos de origen rural, ingresarían alumnos que tuvieran tres años de primaria y se decidió abrir un curso complementario antes de los cuatro años de estudios, para cubrir los años faltantes de la primaria. Las escuelas se organizarían como internados mixtos, si bien en un principio se contempló una proporción baja de mujeres. Todos los estudiantes recibirían una beca del gobierno federal, ya que no se aceptarían alumnos con recursos para costearse su educación.

Además de la educación, las escuelas debían promover el desarrollo económico, cultural y social de las comunidades. Las actividades hacia la comunidad no debían regirse sólo por la buena intención de los maestros sino por el conocimiento de las características y necesidades de las regiones cercanas. Las escuelas se establecerían según regiones geográficas y adaptarían sus planes de estudio y trabajos según dichas características, contarían con campos de experimentación y, al igual que en el proyecto original de las escuelas centrales agrícolas, contarían con la representación del Banco Nacional de Crédito Agrícola. Ello garantizaría que créditos y asesoría técnica fueran de la mano y resultaran más fructíferos que aplicando fórmulas generales.

6 La economía doméstica que se enseñaría en las escuelas regionales campesinas era muy distinta a la desarrollada en el siglo XIX o de las labores femeniles que realizaban las mujeres en las misiones culturales y escuelas normales regionales en los años veinte. Si bien se compartía el patrón de incentivar la economía familiar y modernizar el hogar campesino, la forma y actividades precisas se apegaron mucho más a la vida rural, apartándose aunque parcialmente de patrones urbanos de clase media.

7 SEP, 1933b, p. 69; SEP, 1940a; SEP, 1941; García Téllez, 1935. 
Las escuelas contarían también con Institutos de Acción Social, encargados del mejoramiento cultural y educativo de las comunidades, que también tendrían que sustentarse en estudios específicos, los mismos que realizarían los Institutos de Investigación Social. Todos los estudiantes serían involucrados en las actividades de estas agencias para estar constantemente ligados a la vida de las comunidades y cada regional tendría a su cargo las escuelas primarias de su zona de influencia para que su labor educativa tuviera más firmeza, es decir, los directores de la regional campesina se encargarían de la supervisión de las escuelas, así como de los cursos de mejoramiento para maestros rurales en servicio.

En suma, la idea central era crear un desarrollo integral: el trabajo hacia los campesinos por medio de cooperativas tendría como base la experimentación agrícola que se desarrollara en las escuelas y contaría con el respaldo de los créditos aportados por el banco y la formación de los campesinos en las escuelas rurales, que estarían bajo el control de las regionales. A su vez, la experimentación agrícola y la formación de los estudiantes se retroalimentaría con la participación directa de éstos en el trabajo directo con los campesinos y en todas las actividades escolares.

Este proyecto, sin embargo, se fue reduciendo por diversos motivos. Uno de los principales fue que la SAF no quería perder su poder en el otorgamiento de créditos, así es que a raíz de la expedición del nuevo código agrario en 1934, los directores de las regionales dejaron de ser los representantes del Banco Nacional de Crédito Agrícola. A finales de ese mismo año, las misiones culturales también fueron separadas de las regionales campesinas para volver a ser itinerantes, ya que las escuelas regionales no podían abarcar tantas zonas para capacitar, así es que el trabajo de investigación y acción social se encomendó a los maestros y estudiantes. Por otro lado, la ubicación geográfica de las escuelas dependió de negociaciones políticas más que de criterios científicos, aunque dejaron de concentrarse en el centro del país. El sistema creció y se llegó a tener 35 escuelas en 1936, pero la expansión no contó con un presupuesto adecuado y la mayor parte de los planteles de nueva creación estaban en las mismas condiciones de pobreza que las primeras normales rurales, pero ahora con más responsabilidades, y muchas no contaban con una plantilla completa de profesores. ${ }^{8}$ En las pequeñas, a veces sólo se impartía el año complementario, y sólo había un maestro trabajando con un grupo de 20 a 50 estudiantes, mientras que en la

8 AHSEP, DEANR, Circulares, 344.15 y 1658/52. Del total de escuelas en 1936, 11 escuelas eran grandes, 11 medianas y 12 muy pequeñas. 
mayoría de las grandes, que podían tener hasta cerca de 400 alumnos y una decena de maestros, el plan de estudios se redujo a tres años. ${ }^{9}$

Con la llegada de Lázaro Cárdenas a la Presidencia, la SEP siguió incentivando el papel que el maestro rural debía realizar en las comunidades para promover el desarrollo económico, pero más que un "ejidatario modelo", se quería formar un maestro rural que, además de capacitación técnica y pedagógica, tuviera "una ideología social íntimamente vinculada con la suerte, con los afanes de lucha, con los problemas de emancipación de la clase campesina". ${ }^{10}$ Las autoridades buscaron reforzar la ideología socialista y que se profundizara "el conocimiento científico de las formas de explotación del hombre sobre el hombre y sobre métodos de lucha social" en las escuelas regionales campesinas y añadieron al plan de estudios de 1935 materias como "conocimiento de los problemas que afectan la vida del campesino mexicano y crítica de las soluciones dadas a la luz de las ideas socialistas", "concepto general del socialismo", "economía e historia del movimiento obrero nacional e internacional", "origen de las religiones y del cooperativismo". Asimismo, dieron prioridad al conocimiento de la Ley Federal del Trabajo, el Código Agrario, la reforma del Artículo Tercero y de la "doctrina marxista" a través de pláticas y conferencias."

Independientemente de las diferencias, el discurso de la educación socialista, exaltando los intereses colectivos sobre los individuales, la emancipación de los trabajadores, la redistribución de la riqueza, la igualdad de los derechos, el cooperativismo y la co-educación, daba coherencia al trabajo que en muchas de estas escuelas se venía realizando desde años atrás con los campesinos como la formación de cooperativas y la lucha contra los acaparadores. La educación socialista fue una retórica que cobijó prácticas que se realizaban desde antes, pero impulsó en los estudiantes una identidad colectiva basada en el origen social, ya que para 1936 se había logrado que los alumnos de las escuelas fueran en su mayoría hijos de campesinos pobres $y$, en especial, de ejidatarios. ${ }^{12}$

9 Hubo cuatro categorías de Escuelas Regionales Campesinas. Para un mayor acercamiento al tipo de escuelas, su ubicación geográfica y los criterios para fundar escuelas véase Civera, 2008, pp. 139-156.

10 García Téllez, 1935, p. 230.

11 AHSEP, DEANR, 3015, 16-1-2-100, "Informe general de la Sección Técnica de Acción Social del DEANR", 29 de diciembre de 1936. Véase también SEP, 1940b, y Civera, 1997, pp. 73-88.

12 Los registros no solían especificar si los estudiantes eran indígenas o no. En algunas se reportaban problemas con estudiantes que no hablaban el español, pero por la ubicación geográfica de muchas de ellas podemos suponer que su presencia era importante. 


\section{El impulso cooperativista}

El cooperativismo fue uno de los ejes de las actividades de las escuelas en estos años y los posteriores. ${ }^{13}$ No todos los maestros de las escuelas regionales campesinas estuvieron de acuerdo con el fomento de la lucha de clases, sin embargo, tanto para los defensores de la escuela vital, ${ }^{14}$ como para los partidarios de la educación socialista, el cooperativismo era importante. También los agrónomos tenían distintas concepciones del este sistema. Para algunos, era una herramienta técnica destinada a elevar la productividad. Julián Rodríguez Adame, un agrónomo influyente en el sector, explicaba en una conferencia que el ejido podía reproducir el sistema explotador de la hacienda si en él prevalecía la producción individual. Al unir esfuerzos, considerar la comercialización y producir colectivamente, las cooperativas podrían realizar una explotación más racional sin contemplar el crédito ejidal "porque este no puede alcanzar más que a una quinta parte de la población". ${ }^{15}$ En 1937, Narciso Bassols hizo una fuerte crítica al proyecto de Ley de Cooperativas elaborado por el poder ejecutivo al que tildaba de engaño, argumentando que el destino final del proletariado no dependía de la organización de un sistema cooperativo, sino que estaba vinculado

a una idea mucho más importante: la de la transformación completa del régimen de propiedad existente. Mientras no se llega a ella, las cooperativas sólo pueden servir como auxiliares y órganos de moderación de los abusos. Pero no debe consentirse en que hasta este atributo se les quite, bajo la ilusión falaz de lograr una prosperidad general basada en la cooperación. ${ }^{16}$

Bassols criticaba la postura gubernamental que concebía a las cooperativas como el puente entre el capitalismo y una sociedad sin clases y que fue compartida por algunos agrónomos, como Mario. F. Morineau, director interino de la escuela regional campesina de Tenería, Estado de México.

13 Desde luego el cooperativismo fue impulsado desde antes, pero en los años treinta tuvo un peso más fuerte en el sistema educativo, particularmente en la enseñanza normal y agrícola.

14 Los maestros pensaban que la educación debía apegarse a la vida, alejándose de la memoria y el verbalismo. La observación y la educación práctica y aplicada se consideraban centrales para orientar un trabajo escolar que debía responder a las necesidades de la gente. Se trataba de una apropiación muy especial de la escuela de la acción de John Dewey y otras pedagogías centradas en el niño.

15 LAS, s/f, p. 20. Bassols dejó de ser secretario de Educación pública antes de que se iniciara el periodo presidencial de Cárdenas, pero su proyecto era compartido por los agrónomos involucrados en el DEANR.

16 Bassols, 1964, pp. 448. 
Morineau escribía en sus informes que constantemente hacía saber a los alumnos que

la única forma de que podrían progresar los campesinos y quitar de una manera definitiva al capitalista del campo, es cuando la tierra fuera explotada por medio de cooperativas aprovechando en ellas la especialisación (sic) de los trabajadores. ${ }^{17}$

A finales de 1934 se aprobó un reglamento para las cooperativas de todas las escuelas federales de distintos tipos que consideraba que el cooperativismo "crea en el niño un amplio sentido de solidaridad y asociación en el esfuerzo, le educa para la producción colectivizada, y le proporciona las posibilidades de disfrutar íntegramente de los beneficios de la vida social". ${ }^{18}$

En octubre de 1936, el secretario de Educación Pública ordenó el establecimiento de una sección de Sociedades Cooperativas en el DEANR para hacerse cargo de la organización, control y fomento de todas las cooperativas escolares. Pero dentro de la SEP y del mismo Departamento no había consenso ni claridad acerca de los fines y métodos del cooperativismo, que igual podía verse como substitución del capitalismo, como paso del capitalismo al socialismo, o como paliativo del capitalismo que sin embargo no debía poner en riesgo la libre competencia. ${ }^{19} \mathrm{La}$ variedad de concepciones acerca del cooperativismo es evidente: se le veía como puente para terminar con las diferencias de clases, medio para evitar el abuso o, como escribirían en 1935 los estudiantes de la escuela regional campesina de Tenería, Estado de México, como medio para lograr "un progreso común", una "vida más justa y equitativa", "la fraternidad", el "mejoramiento de la vida social", "una felicidad igualitaria" o incluso "una paz universal". Pese a esta variedad de interpretaciones, había consenso en que era necesario impulsar el cooperativismo entre los campesinos, y que para ello los estudiantes debían aprender sobre él, no sólo teóricamente, sino en la práctica misma. ${ }^{20}$

17 AHSEP, DEANR, Estado de México, 370.40, Informe del especialista de ganadería, Tenería, 26 de diciembre de 1934.

18 SEP, 1935, p. 163.

19 AHSEP, DEANR, 3012, 16-1-1-222, "Borrador de oficio dirigido a los jefes de misiones culturales, organizadores rurales de las misiones culturales y directores de las escuelas regionales campesinas", 8 de octubre de 1936.

20 Los entrecomillados corresponden a diferentes artículos publicados por los alumnos en la revista Impulso Juvenil, de abril y mayo de 1935. 
En suma, el cooperativismo era considerado como una herramienta imprescindible para luchar en contra del individualismo y el egoísmo, y para matizar, si no remediar, las condiciones de pobreza de los campesinos y los obreros. El vínculo entre la civilización del campesino, la modernidad productiva y la emancipación de las masas era un cooperativismo que aunque buscaba diferentes objetivos, proponía métodos similares de organización social y se colocaba como prerrequisito para mejorar las condiciones de los campesinos e incluso dirigirlos hacia su liberación. Se trataba de un concepto cuya ambivalencia se derivaba de la propia indefinición del ejido como figura jurídica que ponía en tensión los afanes socialistas con el modelo capitalista que la Revolución buscaba impulsar.

Sin embargo, una cosa era lo que se quería impulsar y otra muy distinta la que se enseñaba día a día en las escuelas, en cooperativas cuyo funcionamiento fue muy problemático.

\section{Las cooperativas de las escuelas regionales campesinas}

Todas las escuelas regionales campesinas debían organizar sus cooperativas de consumo y producción. ${ }^{21}$ Para 1936, ya todas las escuelas contaban con algunas, pero funcionaban con fuertes deficiencias: se llevaba mal la contabilidad, o se contabilizaban recursos de la escuela que no debían contemplarse, o no todos los estudiantes participaban en ellas. ${ }^{22} \mathrm{~A}$ principios de 1937, el DEANR intentaba aún que los estudiantes tuvieran el control de las de alimentación (lo cual implicaba el control sobre las becas) y que los directores sólo fueran asesores. Aunque las becas eran bajas, algunas cooperativas lograban generar ahorros, sin embargo ello no se veía como un beneficio, ya que la SEP ordenó que los ahorros no podían repartirse entre sus integrantes. ${ }^{23}$

21 Su objetivo era administrar las becas de los estudiantes y las cuotas de los maestros para tener una buena alimentación en el internado a un bajo costo y generar ahorros. Supuestamente debía de ser manejada por los estudiantes guiados por la maestra ecónoma, para que ellos compraran los alimentos aprendiendo a balancear los nutrientes, supieran elaborarlos, sirvieran las mesas y limpiaran el comedor. Además debían llevar las cuentas, con lo cual practicaban también matemáticas.

22 Para más detalle véase Civera, 2008, pp. 187-206.

23 AHSEP, DEANR-Subsecretaría, 3081, 16-3-8-171, "Informe de las labores desarrolladas por la Sección de Cooperativas, durante el periodo comprendido entre el primero de septiembre de 1936 y el último de agosto del año actual". 
Desde 1933, Mesa Andraca había tratado de reglamentar las sociedades de producción escolares. Al hablar sobre la enseñanza agrícola en las escuelas, decía que debía tomarse en cuenta

el propósito de educar a los alumnos a base del trabajo en cooperación y colectivo, no
en forma individual, lo cual sólo puede ser útil para muy especiales enseñanzas. Hay
que intentar demostrar siempre que la división del trabajo y la cooperación efectiva
de un grupo determina una realización más adecuada y un rendimiento más alto en
las explotaciones..$^{24}$

Las cooperativas podrían proporcionar a los estudiantes un pequeño fondo para establecerse como pequeños agricultores al acabar sus estudios y, siempre que fuese posible, debían incluir a los estudiantes y a los campesinos que trabajaran para las escuelas. En 1934 el ingeniero Julián Rodríguez Adame, director de Tenería, elaboró un proyecto de reglamento general para su funcionamiento. En él se aclaraba que el fin de éstas era enseñar la técnica agrícola, ganadera, industrial y de oficios realizando una intensa práctica dentro del ambiente natural; "enseñar prácticamente la economía rural, determinando todos los factores y elementos que rigen los costos, valores de los productos, utilidades o pérdidas, haciendo de la contabilidad rural, de los registros, las gráficas y cuadros comparativos imprescindibles y poderosos auxiliares para orientar todas las actividades, que como la agricultura, fundamentalmente constituyen un negocio"; formar "la conciencia de clase para la juventud rural" y que la vida escolar rural se realice a semejanza de su medio, preparando a la juventud para mejorarla en beneficio de los intereses colectivos" ${ }^{25}$.

En 1936 este proyecto, con algunas modificaciones, era el que se enviaba a las escuelas regionales campesinas para reglamentar las cooperativas. Pese a los lineamientos de cooperativas escolares de 1934 y 1936, en las regionales aún en ese último año cada escuela elaboró sus propias bases de funcionamiento. Todos los planteles tenían sociedades de producción agrícola, pero pocos tenían de industrias, conservación de frutas y legumbres, herrería, carpintería, ganadería, cajas de ahorro, molinos de nixtamal y de fomento forestal. Las variables condiciones de las escuelas, así como la falta de recursos para contratar los ingenieros agrícolas que hacían falta,

24 SEP, 1933b, pp. 176.

25 AHSEP, DEANR, 3012, 16-1-1-223, "Proyecto para la enseñanza y organización cooperativa en las escuelas del Departamento de Enseñanza Agrícola y Normal Rural", 16 de diciembre de 1935. 
hicieron que en las escuelas pequeñas este tipo de enseñanza fuera muy elemental. Incluso en las grandes, por la falta de ingenieros agrónomos preparados y de recursos, la enseñanza agrícola no era muy eficiente.

A finales de 1936, los miembros de la Liga Socialista de Agrónomos, entre ellos Manuel Mesa Andraca, dieron una serie de conferencias con las que pretendían convencer a las autoridades para que devolvieran a las regionales su proyecto original, orientado no sólo a la formación de maestros rurales sino también de técnicos agrícolas con espíritu social, capacitados en técnicas modernas de producción. Para ello, decían, era imprescindible otorgar a los planteles recursos para experimentación y demostración, porque en las escuelas se cultivaba "sin mayor chiste". ${ }^{26}$ Para 1940, la mayor parte de la producción de estos centros era de maíz, trigo, alfalfa, garbanzo, frutas y verduras, y tenían ganado ovino, caprino, vacuno y porcino. ${ }^{27}$ Se trataba de producción relacionada con el consumo interno más que externo, y tenía poco que ver con el impulso que se dio durante el cardenismo a la producción colectiva para la exportación de algodón, arroz, café y henequén en regiones como La Laguna, el valle Yaqui, Los Mochis, Lombardía, Nueva Italia, Mexicali, Soconusco y Yucatán.

Las escuelas no tenían la capacidad de desarrollar técnicas modernas, experimentar cultivos y menos de enseñarlos y la SEP tampoco había promovido la capacitación de los profesores en este sentido. Las propias autoridades informaban que las cooperativas de producción de las escuelas no funcionaban mejor por la falta de recursos y sobre todo por la lentitud de la Sección Administrativa para movilizar las partidas. ${ }^{28}$ Además, los agrónomos conseguían otros puestos en la SAF mejor remunerados y de menor esfuerzo que en las escuelas regionales campesinas, así es que para finales de la década la mayor parte de éstas carecía de técnicos. Pese a ello, incluso en las escuelas pequeñas, los maestros intentaron fomentar cooperativas que, más que ser sociedades de tipo económico, eran formas de organizarse en conjunto ayudándose los unos a los otros. ${ }^{29}$

26 LAS, 1936, p. 34.

27 SEP, 1940b, p. 189.

28 AHSEP, DEANR-Subsecretaría, 3081, 16-3-8-171, "Informe de las labores desarrolladas por la Sección de Cooperativas, durante el periodo comprendido entre el primero de septiembre de 1936 y el último de agosto del año actual"; "Informe que se rinde al C. Subsecretario de Educación Pública acerca de lo que se ha venido haciendo en relación con el desarrollo del Plan Mínimo de acción del DEANR durante el primer semestre del año de 1937"; "Informe que rinde la Sección Técnica Agrícola de las labores desarrolladas del primero de septiembre de 1936 al 30 de junio de 1937".

29 AHSEP, DEANR, 3074, 16-3-5-160, "Documentos relacionados con el personal", 1937; Civera, 2008, pp. 164-170; SEP, 1940a. 
Según los informes de las autoridades de la SEP, seis centros estaban en lugares insalubres y no tenían el mínimo de terreno de cultivo. Pero en las escuelas grandes la producción era importante. La de Salaices, Chihuahua, por ejemplo, tenía 1,165 cabezas de ganado y 750 hectáreas de tierra (250 de riego y 500 de temporal); Roque, Guanajuato, contaba con 280 cabezas de ganado y 1,277 hectáreas de tierra (550 de riego y 707 de temporal) ${ }^{30}$ Las cooperativas eran mucho más complejas y probablemente en algunas también había una simulación. ${ }^{31}$ Las sociedades trabajaban por separado y no incluían a todos los estudiantes, ya que su inclusión en una u otra dependía de lo que se les quería enseñar. No todas las tierras de las escuelas se producían en forma cooperativa, porque a veces se dejaban campos para demostración, o se dejaban descansar las tierras. Las que existían eran dirigidas por los maestros especialistas y el director, quien era el responsable ante el DEANR.

En la mayor parte de las sociedades, los socios no invertían, sino que se trabajaba a partir del presupuesto aportado por el Gobierno para las explotaciones y en todas se utilizaban los aperos y máquinas, abonos, semillas y animales de la escuela, sin contabilizar su desgaste o su costo. En Soconusco, Chiapas, los alumnos aportaron gallinas y en otras escuelas los maestros o directores también contribuyeron con herramientas o materiales como en los años veinte, pero estos recursos más bien eran donaciones y no inversiones que debían amortizarse. Tampoco se contabilizaban adecuadamente, según consta en un informe especial de la SEP, los jornales y sobre todo el trabajo de los estudiantes, lo cual causaba, se decía, que ellos no tuviesen mayor interés en la producción. ${ }^{32}$

30 AHSEP, DEANR-Subsecretaría, 3081, 16-3-8-171, "Informe que rinde la Sección Técnica Agrícola de las labores desarrolladas del primero de septiembre de 1936 al 30 de junio de 1937”; Sepúlveda, 1976, p. 150.

31 Todas las escuelas tenían que informar que tenían sociedades cooperativas, pero hay indicios de que algunas, aunque sí las reportaban, no las tenían en realidad, sobre todo las de nueva creación, en las que más bien los vecinos y estudiantes apoyaban la construcción de la escuela y no contaban con tierras ni con animales. Aunque el nombre oficial es "Sociedad Cooperativa", coloquialmente se hablaba de ellas como "sociedades" y sobre todo como "cooperativas".

32 AHSEP, DEANR-Subsecretaría, 3081, 16-3-8-171, "Informe sintético de las anomalías técnico-educacionales más salientes que se observan, contablemente, en los estados anuales de las escuelas regionales campesinas, establecidas en la República", 31 de marzo de 1937 (la firma no es legible); e "Informe que se rinde al C. Subsecretario de Educación Pública acerca de lo que se ha venido haciendo en relación con el desarrollo del Plan Mínimo de acción del DEANR durante el primer semestre del año de 1937'. No hay datos para saber qué tanto los estudiantes eran involucrados o estaban informados de los rendimientos de las cooperativas, si bien, supuestamente, la información debía circular en las asambleas que se debían realizar una vez por mes y si los estudiantes llegaron a obtener ganancias por sus trabajos en las cooperativas. 
A partir de 1936 se simplificó un poco la organización de las cooperativas: todos los alumnos formaban parte de una sola, aunque ésta estuviera compuesta por diferentes cultivos que eran atendidos por los mismos según su grado escolar y los planes de estudio, si bien se seguían separando las sociedades según su tipo: agrícola, ganadera, de industrias, etcétera. Sólo en pocas escuelas se logró formar cooperativas de la caja refaccionaria agrícola e industrial, y no se consiguió tampoco una federación para que las cooperativas de producción de las escuelas pudieran apoyarse unas a otras. Según la SEP, en 1936 todas las escuelas, menos dos, registraron pérdidas. A finales de la década, la SEP decidió centralizar los recursos de las cooperativas escolares, y los estudiantes y maestros reclamaron porque la Secretaría se quedaba con las ganancias que ellos habían generado con su trabajo. Esta medida intensificó, como las mismas autoridades reconocieron, que los estudiantes "con razón se sintieran explotados por el propio gobierno". ${ }^{33}$

Aunque ya no existía la intención de que las escuelas fueran autofinanciables porque, como diría el ingeniero Marco Antonio Durán, era más importante "la utilidad cultural, no la económica", ${ }^{34}$ cabe preguntarse qué tipo de enseñanza recibían los alumnos cuando se registraban frecuentemente pérdidas a pesar del subsidio federal. El fracaso productivo de las escuelas era un factor más que alentaba que los estudiantes buscaran opciones de vida diferentes a las del trabajo agrícola. De hecho, la mayor parte de ellos prefería seguir sus estudios para obtener una plaza como maestro rural, a regresar a sus casas a cultivar las tierras o trabajar como peritos agrícolas para el gobierno. ${ }^{35}$ Ello se relacionaba con la seguridad laboral, pero indudablemente también influía un rechazo al trabajo manual, promovido por la exaltación de la civilización urbana frente al atraso del campo

33 Ibidem, "Informe de las labores desarrolladas por la Sección de Cooperativas, durante el periodo comprendido entre el primero de septiembre de 1936 y el último de agosto del año actual". Véase también Sepúlveda, 1976, pp. 188-200.

34 LAS, 1936, p. 34.

35 AHSEP, DEANR-Subsecretaría, 3081, 16-3-8-171, "Informe sintético de las anomalías técnico-educacionales más salientes que se observan, contablemente, en los estados anuales de las escuelas regionales campesinas, establecidas en la República", 31 de marzo de 1937 (la firma no es legible); "Informe que se rinde al C. Subsecretario de Educación Pública acerca de lo que se ha venido haciendo en relación con el desarrollo del Plan Mínimo de acción del DEANR durante el primer semestre del año de 1937"; e "Informe de las labores desarrolladas por la Sección de Cooperativas, durante el periodo comprendido entre el primero de septiembre de 1936 y el último de agosto del año actual”; Sepúlveda, 1976, pp. 188-200; Civera, 2008, pp. 425-454. 
en la visión de los agrónomos y sobre todo de los maestros. Si en las escuelas, contando con subsidio, herramientas y conocimientos, la tierra no era productiva, más difícil parecía la producción en los ejidos, que pocas veces contaban con estos soportes.

Por otro lado, la SEP reglamentó la forma en que se establecían contratos con peones o aparceros para trabajar las tierras de las escuelas que no podían abarcar los maestros y los estudiantes y a finales de 1935 se comenzaron a organizar cooperativas campesinas o de ex alumnos para las tierras de las escuelas que poseían más de 150 hectáreas. ${ }^{36}$ Asimismo, por un acuerdo presidencial, las escuelas entregaron al Departamento Agrario sus tierras innecesarias para ser repartidas y quedarse con un máximo de 250 hectáreas, que era el límite que podía tener una propiedad según el Código Agrario de 1934. Siete de ellas repartieron sus superficies sobrantes. ${ }^{37}$ Para 1937, según la SEP, ya todos los contratos de aparcería se habían disuelto y se moderaron los proyectos productivos para evitar la contratación de jornaleros. El reparto de tierras, la disminución en la contratación de jornaleros y la desaparición de la aparcería reforzaron el carácter revolucionario y popular de las regionales campesinas, decididas por el ejido y la organización colectiva. Ello contribuyó, junto con el cuidado de que los trabajos fueran intensivos pero no excesivos, a que los estudiantes no vieran como una explotación las labores que tenían que realizar en estos centros, como sucedía antes en las escuelas centrales agrícolas. A principios de 1935 los estudiantes solicitaron que las regionales se sacudieran del sistema de peonaje para trabajar en forma de cooperativismo, considerando que la propiedad privada originaba desigualdades e injusticias y era contraria a los postulados de la educación socialista y del ideario de las regionales campesinas. Pero en años posteriores las autori-

36 Las escuelas llevaban la dirección técnica de la producción y proporcionaban la ayuda necesaria a los cooperativistas quienes, a cambio, pagaban los préstamos y entregaban al almacén escolar el $20 \%$ de sus productos. Además, debían inscribirse como alumnos, aunque en calidad de irregulares. AHSEP, DEANR, 3012: s/n, "Proyecto sobre formación de cooperativas", Tenería, 26 de agosto de 1936, y "Oficio del Jefe del DEANR para confirmar afirmaciones verbales al director de Tenería", 17 de septiembre de 1936.

37 En Tamatán, Tamaulipas, se repartieron 277 hectáreas. Aunque los expertos querían dejar a la escuela entre 115 y 150 hectáreas, se repartieron tierras que eran consideradas como necesarias para la institución, que se quedó con 63. En el caso de El Mexe, algunos de los beneficiados fueron los campesinos que habían trabajado las tierras de la escuela como aparceros o como cooperativistas. Con este reparto las regionales campesinas mejoraron sus relaciones con algunos de los vecinos de los pueblos beneficiados, aunque varias siguieron teniendo problemas por el control del agua. Sepúlveda, 1976, pp. 226-229. 
dades de la SEP manejaban este mismo discurso y ya no fue necesario repetir dicha solicitud..$^{38}$

Como puede verse, las cooperativas de producción escolares rara vez obtuvieron buenos resultados económicos y el entrenamiento de los estudiantes en ellas, tanto en aspectos agropecuarios como de contabilidad y organización, fue deficiente: los métodos de cultivo no eran modernos, el tipo de cultivos tampoco era innovador, si bien las regionales podían aportar a los campesinos la mejora de ganados y semilla, y a veces técnicas para prevenir plagas o enfermedades. Así es que las escuelas no tenían capacidad para apoyar ni la producción ejidal sin créditos, ni la agricultura a gran escala. Ofrecían, en cambio, la enseñanza de técnicas de diversificación de la economía familiar y aprovechamiento de los recursos, como la siembra de huertas, la apicultura, la elaboración de conservas, o los corrales con gallinas, puercos y otros animales. En las cooperativas, además, se confundía quiénes eran los integrantes, quiénes invertían o prestaban, así como quiénes tenían derecho a las ganancias (si se llegaba a tenerlas). Los alumnos, sin embargo, aprendían cooperativismo no sólo dentro de las escuelas.

\section{Las cooperativas agrícolas creadas por las escuelas regionales campesinas}

La familiarización de los estudiantes con el cooperativismo tuvo más fuerza quizás hacia fuera de las escuelas; muchas de ellas volcadas en la organización de las comunidades cercanas con el reparto agrario, además de obtener más adeptos para las escuelas, también provocaron la reacción de los sectores afectados. La mayoría de las regionales, quizás todas, participaron en la organización de los campesinos para obtener tierras, agua y créditos, así como luchar contra los intermediarios. Los directores y maestros hacían partícipes a los alumnos. En Santa Lucía, Durango y Ricardo

38 En 1936 aún se registraba el pago de jornales en algunas cooperativas, pero no es posible saber, a partir de la información disponible, si estos jornales correspondían a peones, a los propios alumnos o a ambos. AHSEP, 509: 4-8-8-30, "Conclusiones del Primer Congreso de Estudiantes Campesinos de la República”, Roque, Guanajuato, 1935; AHSEP, DEANR-Subsecretaría, 3081, 16-38-171, "Informe sintético de las anomalías técnico-educacionales más salientes que se observan, contablemente, en los estados anuales de las escuelas regionales campesinas, establecidas en la República", 31 de marzo de 1937 (la firma no es legible) e "Informe de las labores desarrolladas por la Sección de Cooperativas, durante el periodo comprendido entre el primero de septiembre de 1936 y el último de agosto del año actual". 
Flores Magón, Chihuahua, se crearon formalmente bufetes campesinos, pero aunque no existieran oficialmente, en las regionales les ayudaban con todas las gestiones.

El trabajo político se unía a la asesoría técnica a los ejidatarios, impulsando la producción colectiva y la unión para defenderse de los acaparadores y agiotistas. Las escuelas compartían con los vecinos sus conocimientos agropecuarios y sus recursos (herramientas, sementales, semillas mejoradas, etcétera) si los tenían, pero más que promotoras técnicas de la productividad, su labor era organizadora. En 1937, la SEP buscaba orientar a los agrónomos y les proponía hacer periódicos murales, cursos breves $\mathrm{y}$ trabajar en las parcelas de las escuelas rurales para que sirvieran de campos de demostración y los alentaba a ponerse en contacto con otras instancias gubernamentales para intensificar su trabajo como asesores. En cambio, tan sólo en 1936, las escuelas en su conjunto organizaron formalmente 30 cooperativas de producción y consumo, en algunos casos junto con las misiones culturales. Sin embargo las autoridades de la SEP se quejaban de que eran pocos los directores y maestros que informaban cómo se establecían las cooperativas y en qué condiciones operaban. ${ }^{39}$

En Soconusco, Chiapas, maestros y estudiantes daban pláticas en las comunidades sobre los beneficios del cooperativismo o el primero de mayo sobre la larga lucha del proletariado. Pero en otras escuelas los alcances eran mucho mayores, lo cual los hacía intervenir abiertamente en la política. En Galeana, Nuevo León, los maestros y estudiantes participaban no sólo en las ceremonias de dotación de ejidos, sino que también apoyaban a unos ejidatarios para solicitar ampliación o, junto con el presidente municipal y el secretario del Comité Regional de la Liga de Comunidades Agrarias, trataban de convencer a un grupo de campesinos de que no formaran un "comité blanco" ni compraran tierras. ${ }^{40}$ Dichas gestiones venían de años atrás: el presidente municipal de Galeana había escrito a Narciso Bassols en 1934 para agradecer a los maestros de la escuela por el trabajo social que desarrollaban en apoyo del "elemento agrario". ${ }^{41}$ En los Estados

39 AHSEP, DEANR-Subsecretaría, 3081: 16-3-8-171, "Informe de las labores desarrolladas por la Sección de Cooperativas, durante el periodo comprendido entre el primero de septiembre de 1936 y el último de agosto del año actual"; "Informe que se rinde al C. Subsecretario de Educación Pública acerca de lo que se ha venido haciendo en relación con el desarrollo del Plan Mínimo de acción del DEANR durante el primer semestre del año de 1937”; Sepúlveda, 1976, p. 226.

40 AHSEP, DEANR, 16-1-10-26, Galeana, Informe del director, 14 de mayo de 1936.

41 Ibidem, 344.21, Oficio del presidente municipal al secretario de Educación, 12 de mayo de 1934. 
de México, Sonora, Puebla, Hidalgo, Veracruz y Guerrero también se realizaban trabajos de ese tipo. ${ }^{42}$ En la mayor parte de los casos, se trataba de organizar a los campesinos para luchar por tierras o recursos, más que de la organización de sociedades productivas como tales.

Quizás en forma excepcional, en la escuela de Hecelchakán, Campeche, los alcances políticos fueron de trascendencia, además de los económicos. El director fundador, Juan Pacheco Torres, con los maestros y el apoyo de una Misión Cultural, lograron aglutinar a todos los profesores en el Bloque Revolucionario de Maestros del Estado de Campeche en 1934, organizaron cooperativas ganaderas cuyas actividades amenazaron a los acaparadores y apoyaron a los sindicatos y organizaciones de los marinos y estibadores. Para 1936, estos grupos independientes tenían una gran fuerza en el territorio estatal. El director era acusado de preparar a los niños para la "lucha de agitación anti-patriótica bajo los suspicios (sic) de llamadas doctrinas de una Rusia Soviética" y a los maestros de querer "que nuestra Bandera sea la 'Rojinegra' y que nuestro Himno sea 'La Internacional", La propaganda era impulsada por los carniceros, ya que las cooperativas formadas por los profesores rompieron el poder de su monopolio. $^{43}$

En efecto, en 1935 la segunda guerra cristera ${ }^{44}$ tuvo su mayor apogeo. Los curas hacían proselitismo en contra de la escuela federal y las regionales no fueron una excepción. Entre noviembre de 1935 y agosto de 1936, grupos armados amenazaron a las segundas en Zacatecas, Guerrero, Colima, Guanajuato, Jalisco, Veracruz, Campeche y Oaxaca. A veces el ejército protegía las escuelas, pero generalmente, les daban armas a los maestros y estudiantes para defenderse ellos mismos. En algunas escuelas trasladaban a los estudiantes a otras partes, pero también sucedía que los estudiantes se quedaban para protegerlas. ${ }^{45}$

En la escuela de Ayotzinapa, en 1936, varios estudiantes fueron arrestados y asaltados. La campaña en su contra estaba organizada por un cura

42 Sepúlveda, 1976, pp. 225-226; Civera, 1997; Vaughan, 2000.

43 AHSEP, DEANR, 16-1-5-58, 1936, Estado de Campeche, Personal; AHSEP, Instituto de Orientación Socialista, R-44, 16-21-8-7, 1935 “Cooperativas en el Estado de Campeche”. Véase también Raby, 1974.

44 El gobierno revolucionario desde la década de 1920 desarrolló una política en contra de la Iglesia católica, que tuvo como reacción un movimiento armado de importancia conocido como guerra cristera. En 1934, la modificación del artículo tercero estableciendo la educación socialista hizo que la guerra reviviera, aunque de manera más focalizada.

45 Sepúlveda, 1976, pp. 231-238. 
- cuyo cambio habían solicitado los estudiantes junto con el de dos maestros rurales que eran sus familiares-y por los comerciantes de Tixtla, quienes no estaban de acuerdo en que las tierras del pueblo hubieran sido asignadas a la regional campesina. ${ }^{46}$ En Puebla, el carácter co-educativo alarmaba a los campesinos de la región. En Bimbaletes, Zacatecas, desde finales de 1934 el director de la escuela consultaba con las autoridades de Educación Federal qué podían hacer para terminar con las injurias y vejaciones que sufrían los alumnos por parte de los "fanáticos católicos". El funcionario transcribió el oficio al presidente municipal y, en tono amenazante, advirtió que la escuela sabía cómo defenderse por sí sola pero no lo hacía por respeto a su autoridad. En 1936, los maestros y estudiantes recibieron armas y municiones para protegerla de los grupos alzados. ${ }^{47}$

En Soconusco, Chiapas, aunque aparentemente la escuela no hacía proselitismo contra los curas ni había formado cooperativas, los grupos armados también la atacaron. Los alumnos habían sido rapados por una "epidemia de tiña tonsurante" y en los alrededores había circulado el rumor de que la orden de raparlos vino del gobierno "para bautizarlos nuevamente con el credo socialista". El director estaba ausente en ese momento y sólo los alumnos más jóvenes y las mujeres lograron salir de la escuela en un camión que tomaron por asalto en medio del tiroteo. ${ }^{48}$

En estos conflictos intervenían diferentes tipos de problemas, además de la religión y el enfrentamiento ideológico: las escuelas quedaban en un lugar estratégico entre las comunidades locales y las nuevas organizaciones políticas nacionales. Su intervención en el reparto agrario, la formación de cooperativas y, en muchos casos, su lucha contra los curas, hizo que se ganaran enemigos. Los problemas, sin embargo, se dieron también entre las fuerzas gubernamentales. Dentro del creciente corporativismo estatal, la acción de las regionales campesinas como organizadoras de los trabajadores rurales a veces tomaba sendas independientes que no podía permitir el

46 Ibidem, pp. 234-235.

47 AHSEP, DEANR, 344.17, Escuela Regional Campesina de Bimbaletes, oficios del 10 y 16 de octubre de 1934; Vaughan, 2000, p. 214; Sepúlveda, 1976, p. 233.

48 AHSEP, DEANR, 16-1-10-40, Informe del director Álvaro Narváez;; AHSEP, DEANR, 161-10-40, Internado La Boluda, Chiapas, Informes, 1935-1936. Los estudiantes tenían la costumbre de rapar a los de nuevo ingreso. Dicha práctica se relacionaba con el cuidado de la higiene en los dormitorios, pero también era una forma de distinción social y cultural, como lo hacían notar los grupos atacantes.

49 Sobre todo, no podía permitirse la fuerza del Partido Comunista en la formación del Partido de la Revolución Mexicana (PRM) y la Confederación Nacional Campesina (CNC). 
gobierno federal. ${ }^{49}$ Sin embargo, también jugaban un papel importante las distancias no tan excepcionales entre las comunidades y las autoridades acerca del control de los trabajos pero sobre todo de la posesión de sus bienes materiales.

A principios de 1938, el gobierno de Lázaro Cárdenas expropió la industria petrolera. Además de la escasez de recursos, las presiones internas y externas crecieron y las reformas sociales como la educación socialista y el reparto agrario tuvieron que limitarse. Los trabajos de los maestros y los agrónomos como organizadores rurales comenzaron a ser incómodos para los esfuerzos gubernamentales por encapsular la lucha por el poder en un partido y en el corporativismo estatal. ${ }^{50}$ Las misiones culturales fueron desintegradas y a las escuelas regionales campesinas se les se quitó buena parte del presupuesto que tenían asignado, situación que no cambió a partir de una huelga general organizada en 1940 por los estudiantes, apoyados por sus profesores, en protesta por el abandono de los estudiantes campesinos. En muchas escuelas, sobre todo las medianas, los talleres permanecieron cerrados y las tierras sin trabajar por falta de materiales y de personal. Buena parte de los alumnos tenían materias pendientes ya que no habían podido cursarlas por falta de maestros. Estudiantes y profesores hacían lo que podían para entonces sin el respaldo de las autoridades. ${ }^{51}$ Pero la huelga no triunfó. Durante el gobierno de Manuel Ávila Camacho se reestructuró la SEP desapareciendo el DEANR, separando la formación agrícola de la formación de maestros y a ambas de las escuelas primarias, que comenzaron a trabajar con un plan de estudios uniforme que ya no incluía ni contenidos ni prácticas de agricultura u oficios rurales, ni una orientación cooperativista. Se planteó entonces, enfilándose hacia la modernización, la industrialización y la urbanización en el contexto de la segunda guerra mundial, que la escuela debía ser igual para todos para poder ser realmente democrática. Con ello, la escuela en las zonas rurales perdió el empuje que había tenido desde el término de la Revolución. ${ }^{52}$

Pese a las limitaciones, el radio de influencia que tuvieron las escuelas regionales campesinas fue grande. Orientadas hacia "la organización rural" y la producción para consumo interno, su trabajo - junto con el de las misiones culturales - fueron importantes para fomentar el cooperativismo como forma de organización política más que económica, una forma

50 Explicaciones más amplias al respecto pueden encontrarse en Civera, 2008, pp. 249-257.

51 SEP, 1937; SEP, 1938; SEP, 1940a y SEP, 1940 b.

52 Véase Civera, 2008, pp. 381-432; SEP, 1946 y Greaves, 2008. 
dirigida desde las autoridades gubernamentales y colocada como intermediaria entre éstas y los sujetos políticos locales. Aunque en menor medida, también fueron importantes para mejorar la economía familiar de los campesinos al diversificar las formas de ingreso y maximizar el uso de los recursos materiales y humanos disponibles. La presencia de las misiones y las regionales campesinas era importante a nivel regional y, junto con las inspecciones escolares y las publicaciones de la SEP como El Maestro Rural, en la asesoría y formación del sistema de escuelas rurales que se había formado desde la Revolución.

\section{Consideraciones finales}

En México, la expansión de la escuela a los medios rurales, la creación de un sistema de enseñanza agrícola de corte popular y la integración de contenidos ruralistas en el currículum de la escuela primaria estuvo asociada a la Revolución Mexicana. En un periodo de escasez de recursos económicos, de incertidumbre frente a las tensiones políticas relacionadas con la reforma agraria, así como respecto a las posibilidades de desarrollo económico con base en el ejido, las escuelas rurales y las instituciones ligadas a ellas como las misiones culturales, las escuelas centrales agrícolas y las escuelas regionales campesinas, jugaron un papel fundamental en las vinculaciones de sectores internos de las comunidades con el gobierno federal. En este sentido fue muy importante el cooperativismo, pensado desde diferentes puntos de vista como hemos visto, y aplicado de manera poco exitosa en el ámbito escolar. Este sistema sirvió más como cultura política que como medio para impulsar el desarrollo económico, apoyando el fortalecimiento de un corporativismo estatal que afianzaría su poder central sobre los campesinos alzados en 1910, que no permitió que prosperaran las experiencias en las que la acción de la escuela ponía en peligro dicho control vertical.

A través de las escuelas regionales campesinas podemos ver que la inserción de la enseñanza agrícola en la escuela rural marcó una diferencia sustancial con la escuela pre-revolucionaria, pero su desarrollo fue limitado por varios factores. En los años veinte prevaleció un mayor interés en la alfabetización y el afán de civilizar a los campesinos, pero ni siquiera en los años treinta — cuando se fortaleció la presencia de los agrónomos en la Secretaría de Educación Pública, así como una visión mucho más econo- 
micista y pragmática que intentaba formar un sistema integral del campo enlazando la capacitación y educación (para entonces socialista) con la formación de cooperativas y el otorgamiento de créditos para la producciónse logró que este tipo de enseñanza tuviera un lugar importante para el desarrollo económico del campo. Si no lo fue así en el caso de las regionales campesinas que contaron, aunque con limitaciones, con recursos económicos y humanos, menos lo fue en la escuela rural. El cardenismo impulsó proyectos de producción colectiva con cultivos de exportación en los que la escuela fue importante en el nivel regional, pero en términos generales lo que prevaleció fue una escuela regional campesina que ofrecía una capacitación agrícola que no siempre fue eficiente en su acercamiento a las "necesidades" locales, pero que buscaba atenuar los excesos del capitalismo con una diversificación y mejor organización de los escasos recursos materiales de las familias campesinas, es decir, atenuar la pobreza en una economía familiar-ejidal de subsistencia.

En dicho intento no ayudó, entre otros factores que se han mencionado, una constante tensión entre las posesiones materiales y simbólicas de una institución escolar que se decía federal pero que se sostenía en buena parte en los recursos humanos y materiales de las propias comunidades.

Sin querer minimizar los efectos de la escuela rural revolucionaria, es necesario indicar que, al mediar el siglo XX, sus resultados no habían sido tan efectivos como se esperaba: la desigualdad entre los centros urbanos y rurales siguió siendo muy importante y se intensificaría con el impulso a la industrialización, la urbanización y la escuela democrática unificada de los años cuarenta.

Recibido el 14 de mayo de 2010 Aceptado el 13 de septiembre de 2010

\section{Bibliografía}

Armando Bartra: Estructura agraria y clases sociales en México, México, ERA, 1985.

Narciso Bassols: Obras, México, FCE, 1964.

Alicia Civera: Entre surcos y letras. Educación para campesinos en los años treinta, México, El Colegio Mexiquense, Instituto Nacional de Estudios Históricos de la Revolución Mexicana, 1997. 
- "Entre el campo y la ciudad: la formación de maestros normalista rurales y técnicos agrícolas en México, 1921-1945”, en Flavia Werle (Org.): Educaçao rural em perpectiva internacional, Ijuí, Rio Grande do Sul, Editora Unijuí, 2007, pp. 425-454.

- La escuela como opción de vida: la formación de maestros normalistas rurales en México, 1921-1945, México, El Colegio Mexiquense, 2008.

Joseph Cotter: Troubled Harvest: Agronomy and Revolution in Mexico, 18802002, Westport, Conn, Praeger, 2003.

Ignacio García Téllez: Socialización de la cultura. Seis meses de acción educativa, México, SEP, 1935.

Cecilia Greaves: Del radicalismo a la unidad nacional. Una visión de la educación en el México contemporáneo, 1940-1964, México, El Colegio de México, 2008.

Impulso Juvenil, Órgano mensual de la Escuela Regional Campesina de Tenería, abril y mayo de 1935 .

Liga de Agrónomos Socialistas (LAS): Bases generales y estatutos, México, LAS, 1935.

- Ponencias que presenta la Liga de Agrónomos Socialistas ante el Congreso de Unificación Proletaria, México, LAS, s/f.

- Problemas de la enseñanza agrícola, México, LAS, 1936.

Engracia Loyo: Gobiernos revolucionarios y educación popular en México, 1911 1928, México, El Colegio de México, 1999.

- “Escuelas o empresas? Las centrales agrícolas y las regionales campesinas (1926-1934)”, Estudios Mexicanos, 20-1, México, 2004, pp. 69-98.

Susana Quintanilla y Mary Kay Vaughan (coords.): Escuela y sociedad en el periodo cardenista, México, FCE, 1997.

David Raby: Educación y revolución social en México (1921-1940), México, SEPSetentas, 1974.

Secretaría de Agricultura y Fomento (SAF): Escuela Central Agrícola de Michoacán, México, Dirección de Estudios Geográficos y Climatológicos, 1926.

Secretaría de Educación Pública (SEP): El esfuerzo educativo en México, México, SEP, tomo I, 1928a.

- Las Misiones Culturales en 1927. Las Escuelas Normales Rurales, México, SEP, 1928b.

- Las misiones culturales, México, SEP, 1933a.

- Memoria relativa al estado que guarda el ramo de educación pública el 31 de agosto de 1933, México, SEP, 1933b, tomo II.

- Memoria relativa al estado que guarda el ramo de educación pública el 31 de agosto de 1935, México, SEP, 1935, tomo II.

- Memoria de la Secretaría de Educación Pública, septiembre de 1936-agosto de 1937, México, SEP, 1937, tomo II. 
EL COOPERATIVISMO EN LA ESCUELA RURAL DEL MÉXICO DE LOS AÑOS TREINTA

- Memoria de la Secretaría de Educación Pública, septiembre de 1937-agosto de 1938, México, D.A.P.P, 1938, tomo I.

- Escuelas Regionales Campesinas, México, SEP, 1940a.

- Memoria de la Secretaría de Educación Pública, septiembre de 1939-agosto de 1940, México, D.A.P.P, 1940b, tomo II.

- Memoria de la Secretaría de Educación Pública, septiembre 1940-agosto de 1941, México, SEP, 1941.

- La obra educativa del sexenio 1940-1946, México, SEP, 1946.

Manuela Sepúlveda: La política educativa y las escuelas rurales en la década de los treinta. El caso de las Escuelas Regionales Campesinas en 1936, tesis en Etnología, México, INAH, 1976.

Mary Kay Vaughan: Política Cultural en la Revolución Mexicana: maestros, campesinos y escuelas en México, 1930-1940, México, SEP, 2000. 